\title{
A comparative study of adjustable and non- adjustable sutures in primary horizontal muscle surgery in children
}

\begin{abstract}
Purpose To compare the results of using adjustable and non-adjustable sutures in primary horizontal strabismus surgeries in children.

Methods This randomized control trial included 60 cases of primary horizontal deviation. The adjustable suture (AS) group included 30 patients, and the non-adjustable suture (NAS) group included 30 patients. The follow-up period was at least 6 months. A successful motor outcome was defined as orthophoria or a horizontal tropia of 8 PD or less at both near and far distances. The success rate and ocular drift were recorded and analysed.

Results The mean age in the AS group was $3.48 \pm 2.37$ years at the time of surgery. The mean age in the NAS group was $3.55 \pm 2.64$ years at the time of surgery. The success rate at the end of 6 months was $86.67 \%$ in the AS group and $73.33 \%$ in the NAS group $(P=0.197)$. In exotropic patients, there was a mean undercorrection drift of 2.86 PD in the AS group and a mean undercorrection drift of 2.17 PD in the NAS group. In esotropic patients, there was a mean undercorrection drift of 0.26 PD in the AS group and a mean undercorrection drift of 1.83 PD in the NAS group.

Conclusion There was no significant difference between the groups. However, the success rate was clinically higher in the AS group than in the NAS group.

Eye (2016) 30, 1447-1451; doi:10.1038/eye.2016.144; published online 15 July 2016
\end{abstract}

\section{Introduction}

Adjustable sutures have been popular since the mid-1970s as a technique for improving success

AM Kamal ${ }^{1}$, D Abozeid ${ }^{2}$, Y Seif ${ }^{3}$ and M Hassan ${ }^{3}$

rates after strabismus surgery. ${ }^{1}$ Although most surgeons prefer to use adjustable sutures in recurrent cases in adults, ${ }^{2-7}$ there is no consensus regarding their use in cases involving children. This consensus further decreases with regard to the use of adjustable sutures in primary cases involving children. Although some surgeons use adjustable sutures in all cases of strabismus in children, others do not use adjustable sutures in children at all. This is attributed to a fear of a lack of cooperation, a general inability to examine young children during the recovery period, the increased time and cost required for the surgery and the risks involved in anaesthesia. It has been suggested that the percentage of children who could benefit from the use of adjustable sutures is small relative to the risks involved.

Chan et al, ${ }^{8}$ Dawson et al, ${ }^{9}$ and Engel and Rousta $^{10}$ used an adjustable technique in children with success rates of 74,76 and $88 \%$, respectively. The main disadvantage of these studies was that they did not include a control group and could therefore not confirm that there were improved outcomes in the children who received adjustable sutures $v$ s non-adjustable sutures. Awadein et al ${ }^{11}$ compared the results of using adjustable and fixed sutures in children and reported a success rate of $79 \%$ in the adjustable group vs $64.5 \%$ in the non-adjustable group. Their study was retrospective, and the non-adjustable group of surgeries were performed during an earlier period, whereas the adjustable group of surgeries were performed at a later time.

The aim of this prospective study is to compare results between surgeries in which adjustable sutures and non-adjustable sutures were used in cases of primary strabismus in children.
${ }^{1}$ Faculty of Medicine, Cairo University, Cairo, Egypt

${ }^{2}$ Helwan University, Cairo, Egypt

${ }^{3}$ Faculty of Medicine, Beni Suef University, Beni Suef, Egypt

Correspondence: AM Kamal, Faculty of Medicine, Cairo University, Section 16, Kasr Al-Eini Hospital, Cairo 12311, Egypt

Tel: +201005621723;

Fax: +20233474382.

E-mail: am_oph@yahoo.com

Received: 17 July 2015 Accepted in revised form: 6 June 2016

Published online:

15 July 2016 


\section{Materials and methods}

This study was approved by Cairo University and the Beni Suef University Research Ethics Committee. This is a prospective, comparative, randomized interventional study that was performed on 115 eyes of 60 patients with any type of primary horizontal strabismus. All patients were children. Cases were recruited between June 2012 and June 2014 at our institution. Children who were less than 12 years old presenting with any type of primary horizontal strabismus and were scheduled for surgery were included. Patients with vertical deviations were included, but only the horizontal component was studied. Exclusion criteria included children with restrictive strabismus, paralytic strabismus, Duane syndrome, myasthenia gravis, nystagmus and recurrent strabismus.

Patients were randomly divided using a random number generator into group A and group B. Group A included 57 eyes of 30 patients who underwent horizontal muscle surgery using adjustable sutures (the AS group). Group B included 58 eyes of 30 patients who underwent horizontal muscle surgery using non-adjustable sutures (the NAS group). All patients were followed up for at least 6 months after surgery. Preoperative measurements, the type of strabismus and postoperative results were recorded and analysed. Motor alignment was measured using the alternate cover and prism test at $6 \mathrm{~m}$ for straight, vertical and horizontal gazes and at $0.33 \mathrm{~m}$ for straight gazes. Measurements were recorded with and without correction, and they were repeated twice with tests conducted at least one week apart. In cases in which the angle could not be measured using alternate cover and prism test, the Krimsky test was used. Cycloplegic refraction was performed, and glasses were prescribed for patients with more than 2 diopters of hyperopia or any degree of myopia. Cases with amblyopia were treated before surgery. In both groups, surgery was performed under general inhalational anaesthesia using a fornix conjunctival incision. For recessions, the muscle was hooked and then secured using a double-armed 6-0 absorbable polyglactin 910 suture before disinsertion. In the AS group, an adjustable noose was placed around the muscle sutures. The muscle was held back from the original insertion at the desired amount. A traction suture of 6-0 Vicryl was placed through the sclera to gain access to the adjustable knot during post-op. Adjustable sutures were applied to all recessed muscles. In the NAS group, the recessed muscle was sutured to the sclera after a measurement was made to determine the desired amount of recession. For resections, the desired amount was resected from the muscle, which was secured directly to the sclera in both groups. Non-adjustable sutures were applied to resected muscle. All of the exotropic patients underwent bilateral $\mathrm{LR}$ recession according to a predetermined target angle of deviation except in patients with sensory exotropia. These patients underwent monocular surgery (MR resection, LR recession). Similarly, esotropic patients underwent bilateral MR recession according to a predetermined target angle of deviation except in patients with sensory esotropia, who underwent monocular surgery (LR resection, MR recession) according to surgical tables. In the AS group, children were examined in the recovery room after benoxinate eye drops were applied to facilitate examination. Ocular alignment was assessed at 1-4 $\mathrm{h}$ after surgery using cover-uncover and alternating-cover tests at both far and near distances. At near distances, we used an accommodative target, such as a small picture or a toy. For infants, the Krimsky and Hirschberg tests were used to assay corneal light reflexes. Adjustments were made when needed, the knot was tied, and the noose was removed. Adjustments and the tying of the sutures were performed under intravenous propofol anaesthesia $(2-3 \mathrm{mg} / \mathrm{kg})$. The general goals of adjustments were to leave exotropic patients overcorrected by 6-8 PD at far distances for bilateral recessions and 4-6 PD for recessresect procedures. In esotropic patients, an exotropia of 4 PD was the target in accommodative and congenital esotropia and in orthophoria in sensory esotropia with unilateral recess-resect procedures.

All patients were examined at 1 week and at 1, 3 and 6 months after surgery. A successful motor outcome was defined as orthophoria or a horizontal tropia of 8 PD or less at near and far distances.

Horizontal alignment, ocular drift (the difference between the measurement at 6 months and at 1 week of follow-up) and success rates were recorded and analysed.

\section{Statistical analysis}

All statistical calculations were performed using the computer program SPSS (IBM, United Kingdom). Comparisons of numerical variables between the study groups was performed using Chi square $\left(\chi^{2}\right)$ tests. Fisher's exact test was used when the expected frequency was less than 5 .

\section{Results}

Sixty patients with primary horizontal strabismus were included in the study. Patients were randomly divided into two groups. The adjustable suture (AS) group included 30 patients, and the non-adjustable suture (NAS) group included 30 patients. The mean age in the AS group was $3.48 \pm 2.37$ years at the time of surgery. The mean age in the NAS group was $3.55 \pm 2.64$ years at the time of surgery. The difference in mean age between the groups was statistically non-significant $(P=0.91)$. The difference 
in the preoperative angle between the groups was statistically non-significant $(P=0.9)$. The preoperative data are summarized in Table 1.

\section{Adjustment}

The interval time (the time needed for the patient to regain full consciousness) ranged between 80 and $260 \mathrm{~min}$ with a mean of $156.5 \pm 46.6 \mathrm{~min}$. The methods used in assessments varied according to the level of patient cooperation and included alternate prism and cover tests and Krimsky or Hirshburg tests. Only one child refused to open his eyes for examination among all of the trials, and his sutures were tied. Nineteen patients (66.7\%) reached the target angle without adjustment, and their sutures were tied. Five patients $(16.7 \%)$ were overcorrected and required advancement, and five patients (16.7\%) were undercorrected and required further recession. All cases were adjusted under propofol in the operating room. No complications were recorded during adjustment.

\section{Last follow-up}

The patients included in the study were followed up at 1 week, 1 month, 3 months and 6 months after surgery. The results at 6 months of follow-up are displayed in Table 2. The success rate in the AS group at the end of 6 months was $86.67 \%$ (26 patients). Four patients (13.3\%) in this group were overcorrected, and no cases of undercorrection were recorded in this group. In the NAS group, the success rate at the end of 6 months was $73.33 \%$
(22 patients). One case (3.3\%) was overcorrected, and seven cases $(23.33 \%)$ were undercorrected in this group. The success rate in the AS group was clinically higher than the rate in the NAS group, but the results were statistically non-significant $(P=0.197)$.

In esotropic patients, success was recorded in 22 patients $(87.0 \%)$ in the AS group and in 13 patients $(72.2 \%)$ in the NAS group, which was statistically nonsignificant $(P=0.267)$. Similarly, in exotropic patients, the success rate was also higher in the AS group than in the NAS group (6/7 vs $9 / 12$ patients and 85.7 vs $75.0 \%$, respectively), but this difference was statistically nonsignificant $(P=1)$. Despite the clinically higher success rate observed in the AS group, the statistical nonsignificance can be explained by the small number of patients included in the study.

We next analysed the data regarding changes in ocular alignment and drift at 6 months.

In exotropic patients, in the AS group, no shift was recorded in two cases $(28.6 \%)$, whereas exoshift occurred in four cases $(57.1 \%)$, and esoshift occurred in one case $(14.3 \%)$. In the NAS group, no shift was recorded in seven cases $(58.3 \%)$, exoshift occurred in three cases $(25 \%)$, and esoshift occurred in two cases (16.7\%). In the AS group, the mean drift was $2.86 \pm 4.45 \mathrm{PD}$ of undercorrection (range, -8 to $4 \mathrm{PD}$ ), whereas in the NAS group, the mean drift was $2.17 \pm 5.14$ PD of undercorrection (range, -14 to 4 PD), a difference that resulted in a non-significant $P$-value of 0.359 .

In esotropic patients, in the AS group, no shift occurred in 12 cases $(52.2 \%)$, exoshift was observed in four cases

Table 1 Preoperative data

\begin{tabular}{|c|c|c|c|c|}
\hline & Age( years) & $\begin{array}{l}\text { Gender } \\
M \text { male, F female }\end{array}$ & Preop angle far $(P D)(D c c)$ & Preop angle near (PD) (Ncc) \\
\hline Non-adjustable suture & Mean $=3.55 \pm 2.64(0.5$ to 11$)$ & $M=15 ; F=15$ & Mean $=43.67 \pm 13.70(20$ to 85$)$ & Mean $=42.17 \pm 12.50(20$ to 85$)$ \\
\hline Esotropia 18/30 (60\%) & Mean $=2.38 \pm 1.8(0.5$ to 5.41$)$ & $\mathrm{M}=9 ; \mathrm{F}=9$ & Mean $=45.28 \pm 10.91(30$ to 70$)$ & Mean $=43.61 \pm 8.88(30$ to 70$)$ \\
\hline Exotropia 12/30 (40\%) & Mean $=5.16 \pm 2.83(2$ to 11$)$ & $M=6 ; F=6$ & Mean $=41.25 \pm 17.34(20$ to 85$)$ & Mean $=40.00 \pm 16.79(20$ to 85$)$ \\
\hline Adjustable suture & Mean $=3.48 \pm 2.37(0.6$ to 12$)$ & $\mathrm{M}=14 ; \mathrm{F}=16$ & Mean $=43.37 \pm 10.44(16$ to 65$)$ & Mean $=41.70 \pm 11.16(16$ to 65$)$ \\
\hline Esotropia 23/30 (76.6\%) & Mean $=3.07 \pm 2.25(0.6$ to 12$)$ & $\mathrm{M}=11 ; \mathrm{F}=12$ & Mean $=43.96 \pm 11.36(16$ to 65$)$ & Mean $=41.78 \pm 12.30(16$ to 65$)$ \\
\hline
\end{tabular}

Table 2 Results of 6 months follow-up

\begin{tabular}{lccclll}
\hline & Success rate & Overcorrection & undercorrection & Post-op angle(PD) Ncc $6 m$ & Post-op angle (PD) Dcc 6 $m$ \\
\hline Adjustable suture & $26(86.67 \%)$ & $4(13.33 \%)$ & 0 & Mean $=-0.7 \pm 5.68(-12$ to 15$)$ & Mean $=-1.7 \pm 5.38(-12$ to 12$)$ \\
Esotropia & $20(87.0 \%)$ & $3(13.0 \%)$ & 0 & Mean $=-1.65 \pm 5.13(-12$ to 8$)$ & Mean $=-2.3 \pm 4.83(-15$ to 4$)$ \\
Exotropia & $6(85.7 \%)$ & $1(14.28 \%)$ & 0 & Mean $=2.4 \pm 6.67(-4$ to 15$)$ & Mean $=0.28 \pm 6.96(-6$ to 12$)$ \\
Non-adjustable suture & $22(73.33 \%)$ & $1(3.33 \%)$ & $7(23.33 \%)$ & Mean $=1.46 \pm 8.27(-20$ to 20) & Mean $=0.66 \pm 8.39(-20$ to 20) \\
Esotropia & $13(72.22 \%)$ & $1(5.55 \%)$ & $4(22.22 \%)$ & Mean $=4.1 \pm 8.69(-20$ to 20) & Mean $=3.33 \pm 8.75(-20$ to 20) \\
Exotropia & $9(75.0 \%)$ & 0 & $3(25 \%)$ & Mean $=-2.5 \pm 5.91(-12$ to 6) & Mean $=3.33 \pm 6.16(-12$ to 6) \\
\hline
\end{tabular}

$(-)$ sign indicates exotropic deviation, $(+)$ sign indicates esotropic deviation. 
(17.4\%) and esoshift was observed in seven cases (30.4\%). In the NAS group, no shift occurred in seven cases $(38.9 \%)$, exoshift was observed in three cases $(16.7 \%)$ and esoshift was observed in eight cases (44.4\%). In the AS group, the mean drift was $0.26 \pm 4.6 \mathrm{PD}$ of undercorrection (range, -12 to $14 \mathrm{PD}$ ), whereas in the NAS group, the mean drift was $1.83 \pm 6.96 \mathrm{PD}$ of undercorrection (range, -9 to $20 \mathrm{PD}$ ), a difference that resulted in a non-significant $P$-value of 0.402 .

\section{Discussion}

To our knowledge, this is the first prospective study to compare the use of AS and NAS in children. ${ }^{12}$

The results of our study show statistically nonsignificant differences between these two groups. However, the success rate was clinically higher in the AS group.

In the AS group, $86.7 \%$ of the patients were orthotropic or within 8 PD of orthotropia. These patients were therefore considered successful from a motor aspect. These results are similar to those reported by Engel and Rousta, ${ }^{10}$ who also obtained an $88 \%$ success rate. The current results are also comparable to those of Awadein et $a l^{11}$ and Chan et $a l^{8}{ }^{8}$ who obtained success rates of 79 and $74 \%$, respectively. In the NAS Group, $73.3 \%$ of the patients were orthotropic or within 8 PD of orthotropia. This differs from the $65 \%$ obtained by Awadein et al. ${ }^{11}$ The studies conducted by Chan et $a l^{8}$ and Engel and Rousta ${ }^{10}$ were non-comparative and did not include a control group. We analysed success rates in both esotropic and exotropic patients, and our results were similar. These results were statistically non-significant, which can be explained by the small sample size included in the study.

One of the main obstacles to using adjustable suture in children is the lack of cooperation both during the postoperative evaluation period and during adjustment. Many techniques have been developed to facilitate cooperation in children during examination and manipulation. Chan et $a l^{8}$ performed adjustments on the second day after surgery under topical anaesthesia to obtain better cooperation and to minimize the discomfort and nausea associated with adjustment. Guyton ${ }^{13}$ reported that only four children during his entire career did not open their eyes for examination. His technique entails holding the child in the air upside down and pretending to drop him or her. The child reflexively opens his or her eyes, which allows an assessment of the alignment. In the current study, $96.7 \%$ of the children were cooperative. Alternate prism and cover tests were feasible in $63.3 \%$ of cases, whereas $16.7 \%$ of the patients were examined using the Krimsky test and $16.7 \%$ of them were examined using the Hirshburg test. Only one child was uncooperative despite all attempts to encourage him to open his eyes. To facilitate the examination of children, IV ketorolac was administered before the end of the surgery to relieve pain. We also applied topical benoxinate eye drops to all patients prior to examination, and the examination was performed during the time required for the anaesthesia to wear off (80 to $260 \mathrm{~min}$, with an average of $156.5 \mathrm{~min}$ ). The children were monitored in the recovery room in the company of their parents, who also attended the examination to reassure the child.

To facilitate the performing of manipulations during adjustment, many techniques have been suggested. Chan et $a l^{8}$ avoided the use of a lid speculum and instead allowed the child to experience short periods of rest during adjustment. Dawson et $a l^{9}$ adjusted their patients under topical anaesthesia. Eustis et al ${ }^{14}$ injected subconjunctival lidocaine in children with persistent pain. Engel and Rousta ${ }^{10}$ adjusted the sutures in children at 4-7 h after the primary surgery. They kept the intravenous lines from the surgery in place and allowed patients to imbibe clear liquids until approximately $2 \mathrm{~h}$ before adjustment. Adjustment was performed in the operating room under intravenous propofol sedation or laryngeal mask anaesthesia. Hakim et $a^{15}$ left the releasable suture in place if the alignment was found to be satisfactory on the first postoperative day, but if adjustment was required, lidocaine hydrochloride $2 \%$ jelly was applied to the eye for $5 \mathrm{~min}$, and adjustment was then performed without sedation. Saunders and O'Neil ${ }^{16}$ used a technique to avoid manipulation of the sutures in patients who did not need adjustment. Awadein et al ${ }^{11}$ used either topical proparacaine or intravenous propofol according to the clinical situation and the level of cooperation of the child. The adjustment procedures in our study were performed in the OR within 1-4h of the surgery under a propofol dosage of $2-3 \mathrm{mg} / \mathrm{kg}$ that was administered intravenously through the IV route that was placed earlier for the main surgery. We recommend performing adjustments on the same day as the surgery to avoid the potential healing of the conjunctiva or the adherence of the muscle to the sclera.

In our study, $33.4 \%$ of the patients required adjustment, and $16.7 \%$ were due to overcorrection, whereas $16.7 \%$ were due to undercorrection. Chan et al reported that $27 \%$ of their cases required adjustment, whereas Awadein et al reported a rate of $64 \%$. We could not find a definitive explanation for this variation. Another reason for debate regarding the use of adjustable sutures in children is the inherent uncertainty of the persistence of the alignment that is achieved at the end of adjustment. We observed a tendency towards undercorrection in both exotropic and esotropic patients, irrespective of whether adjustable or 
non-adjustable sutures were used, but this difference was statistically non-significant.

The main drawback of using adjustable sutures in children is the additional time needed for recovery and the post-op examination and adjustment of the sutures. Moreover, the procedure is stressful for the parents and the children, who must continue to fast after the end of the surgery and until the end of the adjustment. All of these drawbacks might be negated if the success of adjustable sutures significantly exceeded that of non-adjustable sutures. In our study, which is the first prospective randomized control trial to test this comparison in selected cases of strabismus (horizontal primary strabismus), we found that the success rate of the AS group was higher than the success rate in the NAS group, but this difference was statistically nonsignificant.

The main weakness of this study is the limited number of included patients (60 patients). We need to recruit a larger number of cases to attain a definitive answer regarding this controversial issue. Another weakness is the lack of proper evaluations of sensory functions of our patients. We were unable to measure the sensory functions of most of our patients because of their young age. We did not use a sample calculation formula. We used a number that was convenient, which is another limitation of the study.

\section{Summary}

What was known before

- Adjustable sutures are considered an alternative technique in adult strabismus surgery.

- No consensus for use of adjustable sutures in children.

- No randomised controlled trials comparing adjustable to non-adjustable sutures for strabismus surgery.

What this study adds

- It is the first prospective comparative study between adjustable suture and nonadjustable suture in children.

- No statistical significant difference between both groups; however, the success rate is clinically higher in adjustable group than non-adjustable group.

\section{Conflict of interest}

The authors declare no conflict of interest.

\section{Acknowledgements}

The authors are grateful to Hala Elhilaly, MD, Professor at the Faculty of Medicine at Cairo University for critically reviewing the manuscript. This research received no specific grant from any funding agency in the public, commercial or not-for-profit sectors.

\section{References}

1 Rosenbaum AL, Metz HS, Carlson M, Jampolsky AJ. Adjustable rectus muscle recession surgery. Arch Ophthalmol 1977; 95: 817-820.

2 Broniarczyk-Loba A, Nowakowska O. Own experience with the use of adjustable sutures in various types of strabismus operations. Klin Oczna 1998; 100(5): 305-309.

3 Eino D, Kraft SP. Postoperative drifts after adjustable-suture strabismus surgery. Can J Ophthalmol 1997; 32(3): 163-169.

4 Rauz S, Govan GA. One stage vertical rectus muscle recession using adjustable sutures under local anaesthesia. Br J Ophthalmol 1996; 80(8): 713-718.

5 Melhuish JA, Kemp EG. The routine use of adjustable sutures in adult strabismus surgery. J R Coll Surg Edinb 1993; 38(3): 134-137.

6 Keech RV, Scott WE, Christensen LE. Adjustable suture strabismus surgery. J Pediatr Ophthalmol Strabismus 1987; 24(2): 97-102.

7 Broniarczyk-Loba A, Nowakowska O, Rozyki A. Adjustable sutures in various types of strabismus operations: surgical techniques. Klin Oczna 1998; 100: 5311-314.

8 Chan TK, Rosenbaum AL, Hall L. The results of adjustable suture technique in pediatric strabismus surgery. Eye 1999; 13: $567-570$.

9 Dawson E, Bentley C, Lee J. Adjustable squint surgery in children. Strabismus 2001; 9: 221-224.

10 Engel JM, Rousta ST. Adjustable sutures in children using a modified technique. J AAPOS 2004; 8: 243-248.

11 Awadein A, Sharma M, Bazemore MG, Saeed HA, Guyton DL. Adjustable suture strabismus surgery in infants and children. J AAPOS 2008; 12: 585-590.

12 Haridas A, Sundaram V. Adjustable versus non-adjustable sutures for strabismus. Cochrane Database Syst Rev 2013; 7: CD004240.

13 Engel JM, Guyton DL, Hunter DG. Adjustable sutures in children. J AAPOS. 2014; 18(3): 278-284.

14 Eustis HS, Elmer TR Jr, Ellis G Jr. Postoperative results of absorbable, subconjunctival adjustable sutures. J AAPOS 2004; 8: 240-242.

15 Hakim OM, El-Hag YG, Haikal MA. Releasable adjustable suture technique for children. J AAPOS 2005; 9: 386-390.

16 Saunders RA, O'Neil JW. Tying the knot: is it always necessary? Arch Ophthalmol 1992; 110: 1318-1321. 